\title{
Differences in Initial Stroke Severity Between Mexican Americans and Non-Hispanic Whites Vary by Age: The Brain Attack Surveillance in Corpus Christi (BASIC) Project
}

\author{
Jeffrey J. Wing ${ }^{\mathrm{a}}$ Jonggyu Baek ${ }^{\mathrm{b}}$ Brisa N. Sánchez ${ }^{\mathrm{b}}$ Lynda D. Lisabeth $^{\mathrm{a}, \mathrm{c}}$ \\ Melinda A. Smith ${ }^{c}$ Lewis B. Morgenstern ${ }^{a, c}$ Darin B. Zahuranec ${ }^{c}$ \\ Departments of a Epidemiology and ${ }^{\mathrm{b}}$ Biostatistics, School of Public Health, University of Michigan, and \\ 'Department of Neurology, Stroke Program, University of Michigan, Ann Arbor, Mich., USA
}

\section{Key Words}

Ethnicity $\cdot$ Severity $\cdot$ Ischemic stroke $\cdot$ Incidence .

Population-based $\cdot$ NIHSS

\begin{abstract}
Background: A wide variety of racial and ethnic disparities in stroke epidemiology and treatment have been reported. Race-ethnic differences in initial stroke severity may be one important determinant of differences in the outcome after stroke. The overall goal of this study was to move beyond ethnic comparisons in the mean or median severity, and instead investigate ethnic differences in the entire distribution of initial stroke severity. Additionally, we investigated whether age modifies the relationship between ethnicity and initial stroke severity as this may be an important determinant of racial differences in the outcome after stroke. Methods: Ischemic stroke cases were identified from the populationbased Brain Attack Surveillance in Corpus Christi (BASIC) project. National Institutes of Health Stroke Scale (NIHSS) was determined from the medical record or abstracted from the chart. Ethnicity was reported as Mexican American (MA) or non-Hispanic white (NHW). Quantile regression was used to model the distribution of NIHSS score by age category
\end{abstract}

$(45-59,60-74,75+)$ to test whether ethnic differences exist over different quantiles of NIHSS (5 percentile increments). Crude models examined the interaction between age category and ethnicity; models were then adjusted for history of stroke/transient ischemic attack, hypertension, atrial fibrillation, coronary artery disease, and diabetes. Results were adjusted for multiple comparisons. Results: There were 4,366 ischemic strokes, with median age 72 (IQR: 61-81), 55\% MA, and median NIHSS of 4 (IQR: 2-8). MAs were younger, more likely to have a history of hypertension and diabetes, but less likely to have atrial fibrillation compared to NHWs. In the crude model, the ethnicity-age interaction was not statistically significant. After adjustment, the ethnicity-age interaction became significant at the 85th and 95th percentiles of NIHSS distribution. MAs in the younger age category (45-59) were significantly less severe by 3 and 6 points on the initial NIHSS than NHWs, at the 85th and 95th percentiles, respectively. However, in the older age category (75+), there was a reversal of this pattern; MAs had more severe strokes than NHWs by about 2 points, though not reaching statistical significance. Conclusions: There was no overall ethnic difference in stroke severity by age in our crude model. However, several potentially important ethnic differences among individuals with the most severe strokes were seen in younger

\section{KARGER}

E-Mail karger@karger.com

www.karger.com/ced (c) 2014 S. Karger AG, Basel

1015-9770/14/0385-0362\$39.50/0
Darin B. Zahuranec, MD, MS

University of Michigan Cardiovascular Center

1500 East Medical Center Drive, SPC \#5855

Ann Arbor, MI 48109-5855 (USA)

E-Mail zdarin@umich.edu 
and older stroke patients that were not explained by traditional risk factors. Age should be considered in future studies when looking at the complex distributional relationship between ethnicity and stroke severity. @ $\quad 02014 \mathrm{~S}$. Karger AG, Basel

\section{Introduction}

A wide variety of racial and ethnic disparities in stroke epidemiology and treatment have been reported $[1,2]$. Race-ethnic differences in initial stroke severity may be one important determinant of racial differences in the outcome after stroke. Previous studies have suggested possible black-white differences in stroke severity, with blacks having more severe strokes than whites [3, 4], but few studies have specifically investigated differences between Mexican Americans (MA) and non-Hispanic whites (NHW). Prior work has suggested no difference in initial stroke severity between MAs and NHWs [5]; however, severity was not the main outcome of interest and this analysis used a simple rank-sum test that may not capture more complex relationships between ethnicity and severity. Other analysis techniques, such as quantile regression, would allow for investigation of ethnic differences on the distribution of severity rather than simply assessing for differences at the mean or median [6]. Quantile regression has been useful in studies of gender differences in thrombolytic therapy for acute myocardial infarction [7] and racial disparities in medication nonadherence [8], both reporting differences that would not have been detected otherwise.

There are several reasons to suspect that complex differences in the distribution of initial stroke severity between MAs and NHWs may be present and benefit from the use of quantile regression. Initial stroke severity, as measured by the National Institutes of Health Stroke Scale (NIHSS) score, is not normally distributed and often skewed. Quantile regression allows for the investigation of potentially heterogeneous effects of ethnicity across the entire range of severity without forcing any assumptions about the distribution of NIHSS. Furthermore, ethnic differences in stroke risk factors might also contribute to differences in severity, with MAs more likely to have diabetes $[5,9]$, and NHWs more likely to have atrial fibrillation (Afib) $[5,10]$. Afib tends to be associated with more severe strokes, which may contribute to an ethnic disparity $[11,12]$. Moreover, age at stroke differs by ethnicity, with MAs generally younger than NHWs [13]. Since stroke severity tends to increase with age, this may also influence ethnic differences in initial stroke severity [14-16].

The overall goal of this study was to move beyond ethnic comparisons in the mean or median severity, and instead investigate ethnic differences in the entire distribution of initial stroke severity. Additionally, we investigated whether age modifies the relationship between ethnicity and initial stroke severity and whether ethnic differences in stroke risk factors accounted for ethnic differences in initial stroke severity.

\section{Methods}

\section{Study Population}

Ischemic strokes were identified from the Brain Attack Surveillance in Corpus Christi (BASIC) project between January 1, 2000 and December 31, 2011. BASIC is an ongoing population-based stroke surveillance study in Nueces County, Texas. Nueces County is located on the Gulf Coast, geographically isolated from the larger cities in southeast Texas, being approximately 150 miles from Houston and San Antonio making complete case capture for stroke possible. Approximately, 340,000 people live in Nueces County with the majority of the population (95\%) residing in the urban city of Corpus Christi. Based on the 2010 Census, $61 \%$ of the population is MA and 33\% is NHW [17]. The methodology of BASIC has been previously described [18]. Briefly, trained abstractors screened ischemic stroke cases from seven hospitals in the county. Out-of-hospital surveillance was included from other sources (emergency departments, neurology offices, nursing homes, office of the medical examiner) at varying times as described previously [18]. Stroke was defined as a focal neurologic deficit of acute onset specifically attributable to cerebrovascular distribution lasting longer than $24 \mathrm{~h}$. Per the BASIC protocol, patients were excluded if they were younger than 45 years, lived outside of Nueces County, or if their strokes were traumatic. We only included an individual's first ischemic stroke during the study time period; this event may have been an incident or recurrent event for the individual. Race/ethnicity was ascertained from the medical record and has been demonstrated to have a 97\% agreement with self-report in the study community (kappa $=0.94)$ [19]. Initial stroke severity was retrospectively calculated with the NIHSS score from data abstracted from the medical record in accordance with a validated method [20] or directly abstracted. Additional information collected from medical records included demographics, history of stroke or transient ischemic attack, traditional stroke risk factors, such as history of stroke/transient ischemic attack (TIA), diabetes, hypertension, Afib, high cholesterol, and heart disease. The BASIC project was approved by the University of Michigan Institutional Review Board and each of the Nueces County hospitals.

\section{Statistical Methods}

Descriptive statistics were calculated both overall and by ethnicity. Categorical variables were compared using chi-square tests and continuous variables were compared using non-parametric Kruskal-Wallis tests. Distributions of NIHSS were examined visu- 
Table 1. Overall demographics and stroke risk factors for ischemic strokes and factors based on ethnicity

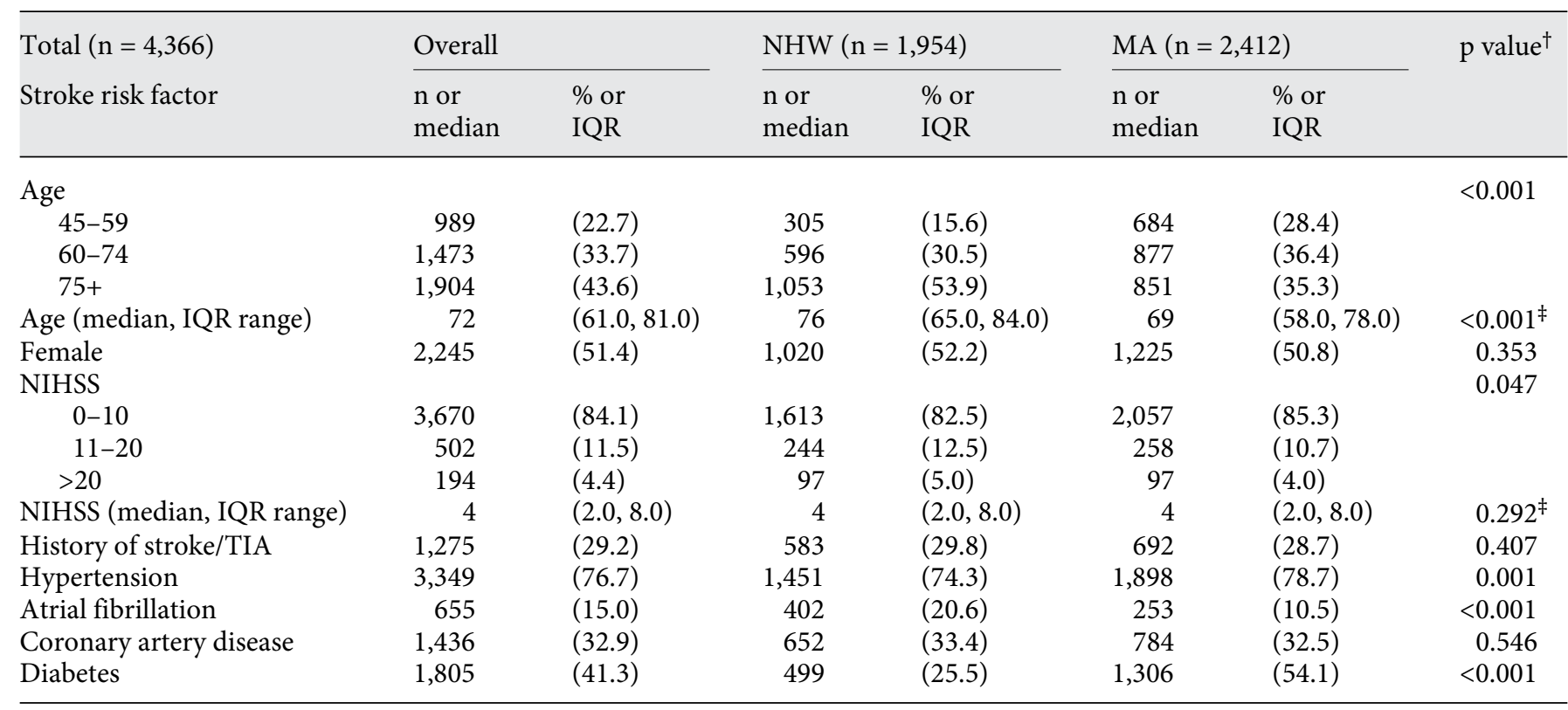

NHW = Non-Hispanic white; MA = Mexican American; IQR = interquartile range; NIHSS = National Institutes of Health Stroke Scale; TIA $=$ transient ischemic attack. ${ }^{\dagger}$ Chi-square test unless otherwise indicated. ${ }^{\ddagger}$ Kruskal-Wallis test.

ally using density plots by ethnicity both overall and by age category (45-59, 60-74, 75+). Quantile regression was used to model the distribution of NIHSS by age category to test whether ethnic differences exist over different quantiles of NIHSS (5 percentile increments). Quantile regression models the conditional percentile of the outcome as a linear function of individual characteristics allowing for the ability to estimate quantile-specific effects at both the median of the distribution as well as any other point through the tails $[21,22]$. This can be thought of as the comparison between the two ethnic groups at a specific point on the distribution, rather than the mean of the distribution as is the case in linear regression. By doing these comparisons across the distribution of initial stroke severity, we can assess where any ethnic differences may occur. Overall quantile regression models were first run to examine the overall relationship between NIHSS and MA ethnicity adjusting for age category. Next, we included an interaction term between MA and age category in the model to examine whether the effect of MA ethnicity varied by age category. Finally, we additionally adjusted for risk factors (history of stroke/TIA, hypertension, Afib, coronary artery disease, and diabetes). Due to multiple hypothesis testing for the interaction effects of MA and age category across 19 quantiles of NIHSS (5-95\%), we adjusted the Type I error rate using an established step-down procedure [23]. All analyses were conducted using the quantreg package of the $\mathrm{R}$ statistical package [24], version 3.0.1.

\section{Sensitivity Analysis}

To investigate the role of NIHSS score calculation method (medical chart or abstractor calculated) in confounding of the ethnicity-age interaction, we created a dummy variable for abstractor calculated NIHSS as well as interactions between the ab- stractor calculated dummy variable and the age category terms. The crude and adjusted models were then replicated including these terms.

\section{Results}

There were 4,366 ischemic strokes identified during the study period (1,954 NHW and 2,412 MA). The median age was 72 years (interquartile range (IQR): 61-81) and median NIHSS score was 4 (IQR: 2-8). MAs were younger, were more likely to have a history of hypertension and diabetes, but were less likely to have Afib compared to NHWs (table 1). MAs and NHWs were similar in terms of prevalence of prior stroke or TIA, coronary artery disease, and high cholesterol.

Visual examination of the overall distribution of NIHSS score demonstrated similar distributions by ethnicity (fig. 1a). Quantile regression demonstrated that there were slight overall differences in the distribution of initial stroke severity by ethnicity in the age category adjusted model; the median of the NIHSS was one point higher among MAs (fig. 1b). These differences were more pronounced within certain age categories. Figure 2 displays the NIHSS distributions by age categories and ethnicity, demonstrating that the distribution of severity was slightly different 


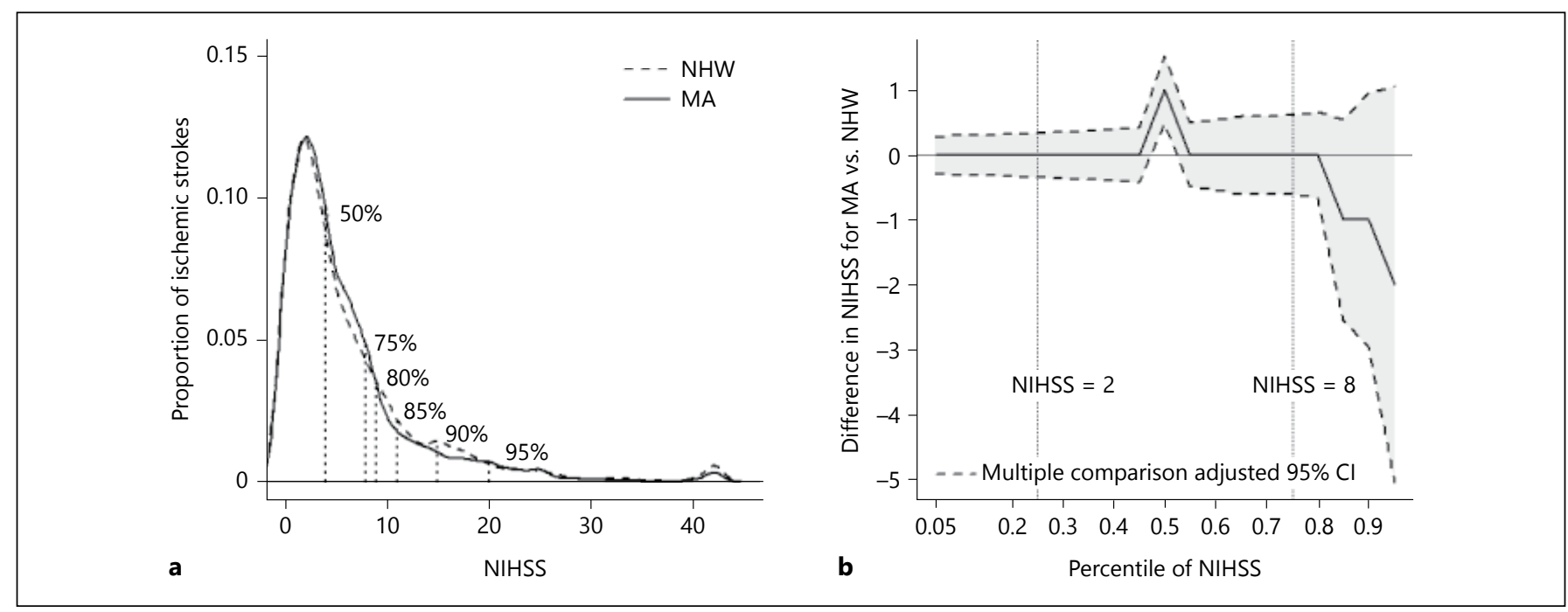

Fig. 1. a Density of the National Institutes of Health Stroke Scale (NIHSS) score by ethnicity among ischemic strokes. NHW indicates non-Hispanic white and MA indicates Mexican American. Vertical lines indicate NIHSS score at the overall percentile. b Ageadjusted ethnic effect (MA vs. NHW) on quantiles of NIHSS score in ischemic stroke, adjusted for age category $(45-59,60-74$, and $75+)$. Vertical lines indicate the 25 th and 75 th percentiles of NIHSS. Dashed lines indicate $95 \%$ confidence intervals (CIs) after multiple hypothesis correction.

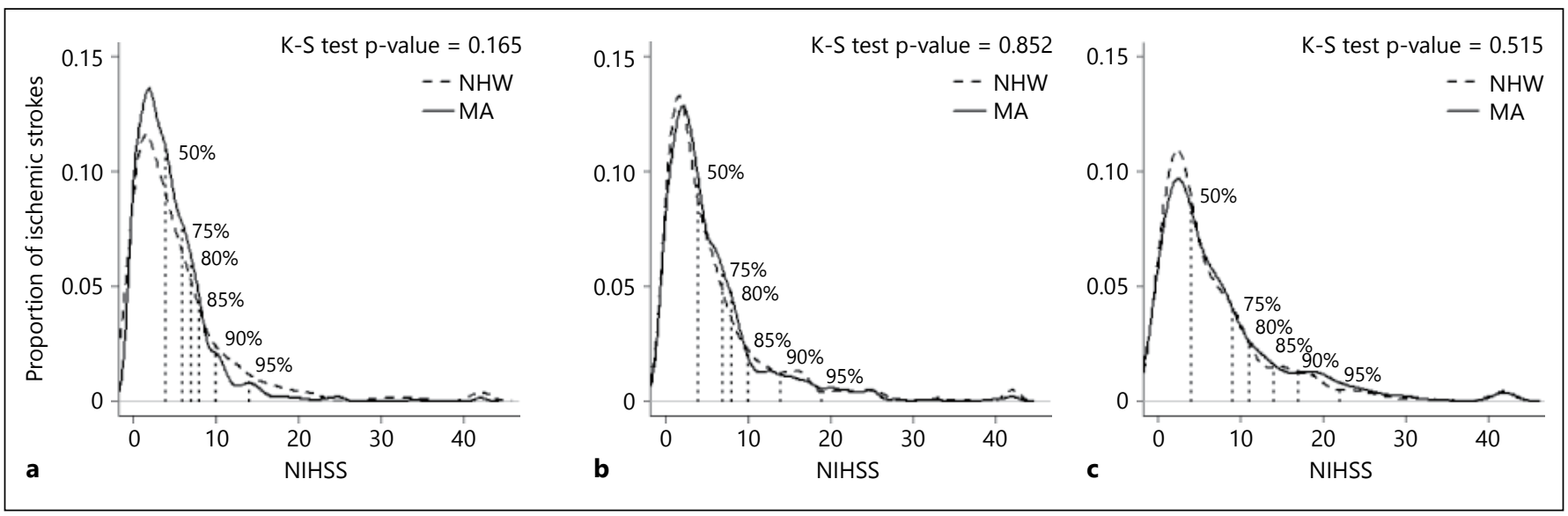

Fig. 2. Distribution of National Institutes of Health Stroke Scale (NIHSS) among ischemic strokes comparing Mexican American (MA) and non-Hispanic white (NHW) by age group: a 45-59, b $60-74$, and c $75+$. The overall percentiles (vertical lines) were included to demonstrate where the differences occur. KolmogorovSmirnov (K-S) tests compared the ethnic distributions. Solid lines indicate MAs and dashed lines indicate NHWs. between ethnicities in the youngest and oldest age groups. When we included the interaction between MA ethnicity and age category in the quantile regression model, the interaction was not statistically significant. We did, however, observe differences in the relationship between ethnicity and NIHSS within some age categories. In the younger (45-59) age category, the 25th and 40th percentiles of NIHSS distribution were one point higher among MAs vs.
NHW, but the 95th percentile was six points lower (fig. 3). In the older (75+) age category, the 30th, 40th, and 50th percentiles of NIHSS distribution were one point higher among MAs compared to NHWs. No severity differences were seen in the 60-74 age category.

After adjusting for potential confounders (history of stroke/TIA, hypertension, Afib, coronary artery disease, and diabetes), the interaction effects between ethnicity 


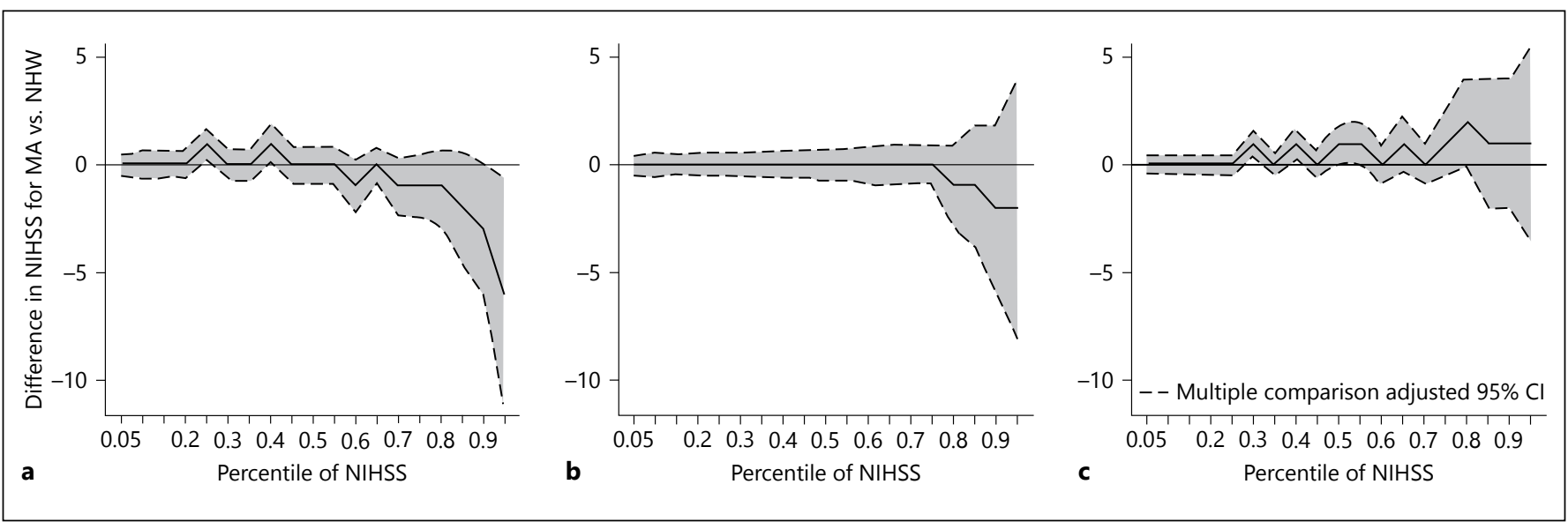

Fig. 3. Age-modified ethnic difference (Mexican American (MA) vs. non-Hispanic white (NHW)) at given percentiles of National Institutes of Health Stroke Scale (NIHSS) score among ischemic stroke patients by age category (a $45-59$, b $60-74$, and c $75+$ ). Dashed lines indicate $95 \%$ confidence intervals (CIs) after multiple hypothesis correction.

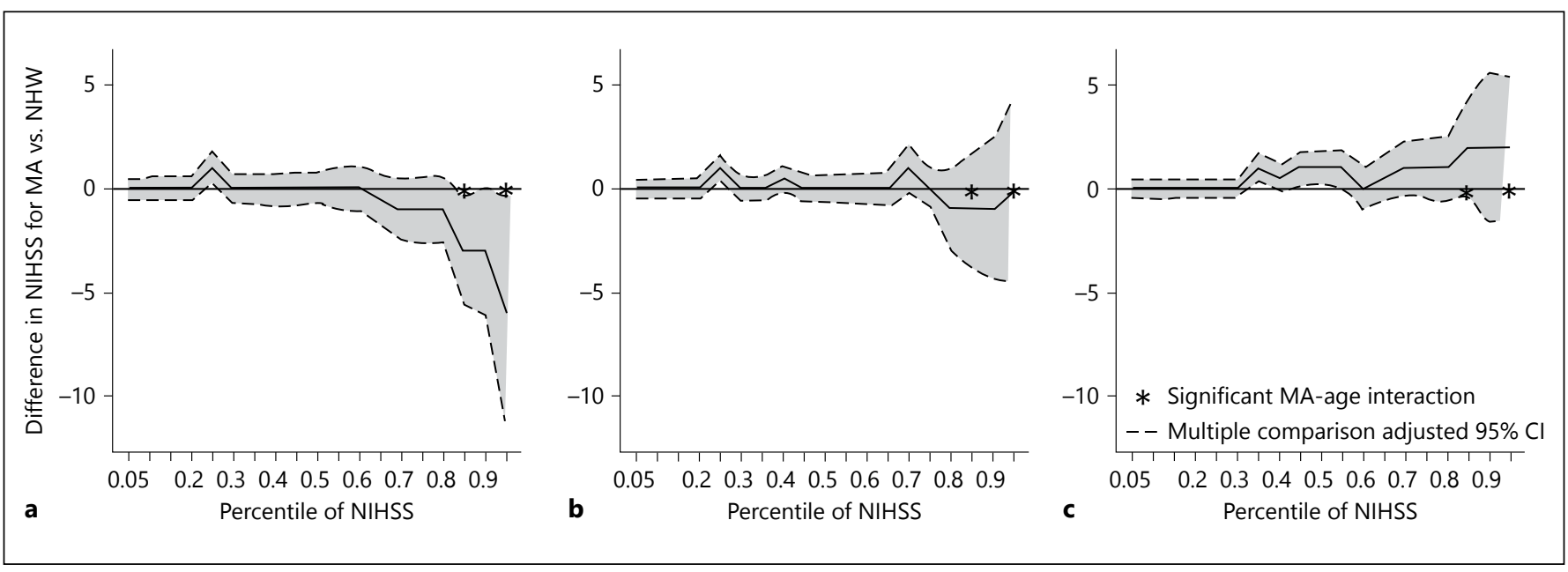

Fig. 4. Fully adjusted ethnic difference (Mexican American (MA) vs. non-Hispanic white (NHW)) at given percentiles of National Institutes of Health Stroke Scale (NIHSS) score among ischemic stroke patients by age category (a 45-59, b 60-74, and c 75+) ad-

and age category became significant at the 85th and 95th percentiles of NIHSS distribution (fig. 4). In the younger age category (45-59), the 85th and 95th percentiles of the NIHSS distribution among MAs were significantly lower than NHWs by 3 points (85th) and 6 points ( 95 th). However, in the older age category (75+), there was a reversal of this pattern, with strokes in MAs being more severe than NHWs by about 2 points, though not significantly. Similar trends could be seen at the lower quantiles after adjusting for potential confounders, but the pattern of justed for hypertension, atrial fibrillation, coronary artery disease, and diabetes. Dashed lines indicate 95\% confidence intervals (CIs) after multiple hypothesis correction. Quantiles that demonstrated a significant ethnicity-age interaction are indicated by a star.

older MAs having more severe strokes at the higher quantiles became more apparent at the 85th percentile.

The results were largely unchanged in the sensitivity analysis incorporating adjustment for confounding by the NIHSS score calculation method. The ethnicity-age interaction in the adjusted models remained significant at the 95th percentile, with MAs being less severe by 6 points compared to NHWs in the younger age category. The ethnicity-age interaction was not significant at any other quantile. 


\section{Discussion}

We observed little overall ethnic difference in initial stroke severity in our age-adjusted model; the median NIHSS score among MAs was only one point higher compared to NHWs. Ethnic differences within the age categories, however, were more apparent. Differences were only seen in the 85th and 95th percentiles of the NIHSS distribution (NIHSS $\geq 8-12$, depending on age). Among the most severe strokes in the youngest age group (45-59 years), MAs had three to six points lower NIHSS scores compared to NHWs. However, at older ages (75+) among the most severe strokes, MAs had two point higher NIHSS scores compared to NHWs.

The implications of severity differences between MAs and NHWs are potentially important for patient care both during the hospitalization and after discharge. Ethnic differences in severity could lead to greater susceptibility to certain complications such as deep vein thrombosis [25] and pneumonia [26]. Severity differences may also impact the burden on stroke family caregivers. MAs tend to prefer family home care/informal care rather than nursing home/formal care $[27,28]$, which could lead to more burden on MA family caregivers given the greater severity seen in older MAs [29].

At younger ages, we found that MAs tended to have less severe stroke compared to NHWs. This might suggest that younger MAs would be expected to have less poststroke disability than NHWs. However, previous studies have found that minorities are less likely than NHWs to return to work after stroke [30] and MAs have worse 90day neurologic, functional and cognitive outcomes [31]. While these studies were not restricted to younger ages, factors beyond the severity of stroke among younger MAs may be responsible for ethnic differences in post-stroke disability and return to work. Further research is needed to better understand the factors in addition to initial stroke severity that may lead to less return to work in younger MAs in order to develop interventions to improve stroke outcomes.

The reasons why younger MAs tend to have less severe stroke than NHWs at the upper quantiles of NIHSS are unclear. However, the ethnic difference is unlikely due to differences in risk factors. In the youngest age group, MAs had lower prevalence of Afib, but had much higher prevalence of both diabetes and hypertension compared to NHWs. Since these risk factors were adjusted for in the models, it is unlikely that they alone are the reason for the disparity. Work from the Greater Cincinnati/Northern Kentucky region has suggested that socioeconomic status

Ethnic Differences in Initial NIHSS Vary by Age contributes to initial stroke severity, with individuals residing in communities of lower SES having greater initial NIHSS compared with those residing in higher SES communities [32]. However, this finding would not seem to explain why MAs have less severe strokes at younger ages at the highest quantiles. Additional research is needed to better understand these differences.

Understanding how severity differs by race and ethnicity has important implications for future research. The existence of a complex relationship between ethnicity and severity suggests that analyses that compare only the median distribution of NIHSS (such as a standard Wilcoxon Rank sum test) may miss important race or ethnic differences in severity. It is important to note that this concern exists primarily when stroke severity is the outcome of interest. However, analyses where NIHSS is a covariate or potential confounder, such as when modeling mortality outcomes, could still be affected by the complex distributional relationship in that incorrect modeling of NIHSS could lead to residual confounding of the exposure effect of interest [33].

The distribution of NIHSS score in our study was similar to other population-based studies. Reeves et al. reported an overall median of 3 with IQR of 1 to 7 [4], which was only slightly lower than our reported $4(2-8)$. The Northern Manhattan Study reported that more than 50\% of ischemic stroke cases had an NIHSS score less than 5 and about $90 \%$ less than 13 [34], compared to $90 \%$ of our strokes having an NIHSS score of less than 15 . This similarity suggests that the associations we found were unlikely to be due to a unique distribution of severity in this community, though it is unknown if similar ethnic differences in severity would be seen in other populations.

Adjustment for known stroke risk factors did not eliminate the ethnic differences in NIHSS, and in fact, the ethnicity-age interaction became significant after adjustment among the most severe strokes. We hypothesized a priori that certain stroke risk factors, in particular Afib and diabetes, might confound the relationship between ethnicity and severity. However, our findings were persistent despite adjusting for these and other risk factors, suggesting that the relationship between severity, ethnicity, and age is not explained by ethnic differences in stroke risk factors.

Our analysis does have limitations. The clinical significance of some of the observed differences may be unclear (e.g., 1-2 points on the NIHSS); however, larger differences were observed in the younger age groups. Despite adjustment for confounders, residual confounding may still exist. For example, while we considered known stroke risk factors, we were not able to adjust for risk fac- 
tor control or certain social or psychological factors such as living alone or dementia, that may be associated with stroke severity [11], and could differ by ethnicity. Additionally, we did not adjust for stroke subtype as we did not have this available for all cases, but we previously did not see ethnic differences in subtype in our population [35]. There could be misclassification of both NIHSS and ethnicity, and the calculation method of NIHSS could influence the results of our analysis; however, our sensitivity analysis found that our results were robust to adjustment of NIHSS score calculation method. The differences we observed in our study population may not translate to other populations with different age and ethnic distributions.

In summary, stroke severity was generally similar between MAs and NHWs, though there were some potentially important ethnic differences among individuals with the most severe strokes. However, these potential ethnic differences in stroke severity were identified in some, but not in all age categories and were not explained by the presence of stroke risk factors alone. Future studies should consider the complex relationship between stroke severity, age, and ethnicity when modeling NIHSS.

\section{Acknowledgments}

None.

\section{Sources of Funding}

Drs. Sánchez, Lisabeth, Smith, and Morgenstern are supported by grant R0138916 from the National Institutes of Health/National Institute of Neurological Disorders and Stroke. Dr. Zahuranec is supported by grant K23AG038731 from the National Institute on Aging.

\section{Disclosure Statement}

None.

\section{References}

1 Cruz-Flores S, Rabinstein A, Biller J, Elkind MS, Griffith P, Gorelick PB, et al: Racial-ethnic disparities in stroke care: the American experience: a statement for healthcare professionals from the American Heart Association/American Stroke Association. Stroke 2011;42:2091-2116.

-2 Towfighi A, Markovic D, Ovbiagele B: Current national patterns of comorbid diabetes among acute ischemic stroke patients. Cerebrovasc Dis 2012;33:411-418.

- 3 Jones MR, Horner RD, Edwards LJ, Hoff J, Armstrong SB, Smith-Hammond CA, et al: Racial variation in initial stroke severity. Stroke 2000;31:563-567.

4 Reeves M, Khoury J, Alwell K, Moomaw C, Flaherty M, Woo D, et al: Distribution of National Institutes of Health stroke scale in the Cincinnati/Northern Kentucky Stroke Study. Stroke 2013;44:3211-3213.

5 Lisabeth LD, Risser JM, Brown DL, Al-Senani F, Uchino K, Smith MA, et al: Stroke burden in Mexican Americans: the impact of mortality following stroke. Ann Epidemiol 2006;16: 33-40.

6 Pourhoseingholi MA, Faghihzadeh S, Habibi $\mathrm{M}$, Safaee A, et al: Association between duration of heartburn and patient characteristics: a quantile regression analysis. Gastroenterol Hepatol 2008;1:71-77.

7 Austin PC, Tu JV, Daly PA, Alter DA: The use of quantile regression in health care research: a case study examining gender differences in the timeliness of thrombolytic therapy. Stat Med 2005;24:791-816.
8 Gebregziabher M, Lynch CP, Mueller M, Gilbert GE, Echols C, Zhao Y, et al: Using quantile regression to investigate racial disparities in medication non-adherence. BMC Med Res Methodol 2011;11:88.

9 Moon JR, Capistrant BD, Kawachi I, Avendaño M, Subramanian SV, Bates LM, et al: Stroke incidence in older US Hispanics: is foreign birth protective? Stroke 2012;43:12241229.

10 Shen AY, Contreras R, Sobnosky S, Shah AI, Ichiuji AM, Jorgensen MB, et al: Racial/ethnic differences in the prevalence of atrial fibrillation among older adults - a cross-sectional study. J Natl Med Assoc 2010;102:906913.

11 Appelros P, Nydevik I, Seiger Å, Terént A: Predictors of severe stroke: influence of preexisting dementia and cardiac disorders. Stroke 2002;33:2357-2362.

12 Kimura K, Minematsu K, Yamaguchi T: Atrial fibrillation as a predictive factor for severe stroke and early death in 15,831 patients with acute ischaemic stroke. J Neurol Neurosurg Psychiatry 2005;76:679-683.

13 Morgenstern LB, Smith MA, Lisabeth LD, Risser JM, Uchino K, Garcia N, et al: Excess stroke in Mexican Americans compared with non-Hispanic Whites: the Brain Attack Surveillance in Corpus Christi Project. Am J Epidemiol 2004;160:376-383.

14 Di Carlo A, Lamassa M, Pracucci G, Basile AM, Trefoloni G, Vanni P, et al: Stroke in the very old : clinical presentation and determinants of 3-month functional outcome: A European per- spective. European BIOMED Study of Stroke Care Group. Stroke 1999;30:2313-2319.

15 Sharma JC, Fletcher S, Vassallo M: Strokes in the elderly - higher acute and 3-month mortality an explanation. Cerebrovasc Dis 1999;9:2-9.

16 Kammersgaard LP, Jørgensen HS, Reith J, Nakayama H, Pedersen PM, Olsen TS: Shortand long-term prognosis for very old stroke patients. The Copenhagen Stroke Study. Age Ageing 2004;33:149-154.

17 US Census Bureau: Census 2010 summary file 2 (SF2). Population of Nueces County, Texas, 2010. Available from www.census.gov [cited October 17, 2012].

18 Morgenstern LB, Smith MA, Sánchez BN, Brown DL, Zahuranec DB, Garcia N, et al: Persistent ischemic stroke disparities despite declining incidence in Mexican Americans. Ann Neurol 2013;74:778-785.

19 Smith MA, Risser JM, Moyé LA, Garcia N, Akiwumi O, Uchino K, et al: Designing multiethnic stroke studies: the Brain Attack Surveillance in Corpus Christi (BASIC) project. Ethn Dis 2004;14:520-526.

20 Williams LS, Yilmaz EY, Lopez-Yunez AM: Retrospective assessment of initial stroke severity with the NIH Stroke Scale. Stroke 2000; 31:858-862.

21 Koenker R, Hallock K: Quantile Regression. J Econ Perspect 2001;51:143-156.

22 Koenker R, Bassett G, Jan N: Regression Quantiles. Econometrica 2007;46:33-50.

23 Liu W: Multiple tests of a non-hierarchical finite family of hypotheses. J R Stat Soc B 1996; 58:455-461. 
24 Koenker R: quantreg: Quantile Regression. R package version 5.05. 2013.

25 Stecker M, Michel K, Antaky K, Cherian S, Koyfmann F: Risk factors for DVT/PE in patients with stroke and intracranial hemorrhage. Open Neurol J 2014;8:1-6.

26 Hoffmann S, Malzahn U, Harms H, Koennecke HC, Berger K, Kalic M, et al: Development of a clinical score (A2DS2) to predict pneumonia in acute ischemic stroke. Stroke 2012;43:2617-2623.

27 Angel JL, Angel RJ, McClellan JL, Markides KS: Nativity, declining health, and preferences in living arrangements among elderly Mexican Americans: implications for longterm care. Gerontologist 1996;36:464-473.

28 Min JW, Barrio C: Cultural values and caregiver preference for Mexican-American and non-Latino White elders. J Cross Cult Gerontol 2009;24:225-239.
29 Luengo-Fernandez R, Gray AM, Rothwell PM: A population-based study of hospital care costs during 5 years after transient ischemic attack and stroke. Stroke 2012;43:33433351.

30 Busch MA, Coshall C, Heuschmann PU, McKevitt C, Wolfe CD: Sociodemographic differences in return to work after stroke: the South London Stroke Register (SLSR). J Neurol Neurosurg Psychiatry 2009;80:888-893.

31 Lisabeth LD, Sánchez BN, Baek J, Skolarus LE, Smith MA, Garcia N, et al: Neurological, functional, and cognitive stroke outcomes in Mexican Americans. Stroke 2014;45:10961101.
32 Kleindorfer D, Lindsell C, Alwell KA, Moomaw CJ, Woo D, Flaherty ML, et al: $\mathrm{Pa}-$ tients living in impoverished areas have more severe ischemic strokes. Stroke 2012;43: 2055-2059.

33 Becher H: The concept of residual confounding in regression models and some applications. Stat Med 1992;11:1747-1758.

34 Dhamoon MS, Moon YP, Paik MC, BodenAlbala B, Rundek T, Sacco RL, et al: Longterm functional recovery after first ischemic stroke: the Northern Manhattan Study. Stroke 2009;40:2805-2811.

35 Uchino K, Risser JM, Smith MA, Moyé LA, Morgenstern LB: Ischemic stroke subtypes among Mexican Americans and non-Hispanic whites: the BASIC Project. Neurology 2004; 63:574-576. 